\title{
LYCHNIS CORONARIA (CARYOPHYLLACEAE) NATURALIZADA EN LA REPÚBLICA ARGENTINA
}

\author{
Rosa L. Scaramuzzino1, Vilma T. Manfreda', Marcelo L. Gandini'1,2 \& Carlos O. D’Alfonso'
}

${ }^{1}$ Facultad de Agronomía, Universidad Nacional del Centro de la Provincia de Buenos Aires, República de Italia 780, 7300 Azul, Buenos Aires, Argentina; rosas@faa.unicen.edu.ar (autor corresponsal).

${ }^{2}$ Comisión Investigaciones Científicas Provincia de Buenos Aires (CIC).

\begin{abstract}
Scaramuzzino, R. L.; V. T. Manfreda, M. L. Gandini \& C. O. D'Alfonso. 2019. Lychnis coronaria (Caryophyllaceae) naturalised in Argentina. Darwiniana, nueva serie 7(1): 179-186.

Lychnis is a genus of native herbs from Eurasia, Northern and Eastern Africa. Many species are cultivated as ornamentals in Argentina, among them L. coronaria. In this paper the presence of a naturalised population of $L$. coronaria is reported for the first time for Argentina. Specimens were collected in the Azul hills, Tandilia System, Buenos Aires province. A description of the morphology of the species and habitat, a map of the collection site, and a germination test based on collected seeds are provided. A key to differentiate Lychnis from its relative genera in Argentina is also provided.
\end{abstract}

Keywords. Alien species; Argentinean Flora; Caryophyllaceae; Lychnis; Tandilia System.

Resumen. Scaramuzzino, R. L.; V. T. Manfreda, M. L. Gandini \& C. O. D'Alfonso. 2019. Lychnis coronaria (Caryophyllaceae) naturalizada en la República Argentina. Darwiniana, nueva serie 7(1): 179-186.

Lychnis es un género de hierbas nativas de Eurasia y el norte y este de África, con algunas especies cultivadas como ornamentales en la Argentina, entre ellas L. coronaria. En este trabajo se informa por primera vez sobre la presencia de una población espontánea de L. coronaria en Argentina. Se recolectaron especímenes en las Sierras de Azul, Sistema de Tandilia, provincia de Buenos Aires. Se aporta una descripción de su morfología y del hábitat y se presenta un mapa del sitio de colección, una valoración de la germinación de las semillas colectadas y una clave para diferenciar el género Lychnis de otros afines representados en la Argentina.

Palabras clave. Especies exóticas; Flora Argentina; Caryophyllaceae; Lychnis; Sistema de Tandilia.

\section{INTRODUCCIÓN}

Caryophyllaceae Juss. es una familia que comprende alrededor de 3000 especies (Rabeler \& Hartman, 2005). Para la flora argentina se consignan actualmente 120 especies entre nativas y adventicias (Flora Argentina, 2019). Asimismo, se cultivan en la Argentina algunos híbridos y al menos 41 especies (Dimitri, 1987), de las cuales algunas se han naturalizado, comoporejemplo,Saponariaofficinalis L.
Una de las especies cultivadas desde hace décadas por su valor ornamental en la Argentina es Lychnis coronaria (L.) Desr. (Dimitri, 1959), considerada perenne de vida corta o bianual según las condiciones de cultivo (Hartman \& Kester, 1986), con requerimiento obligatorio de vernalización para su reproducción sexual (Chouard, 1960). Es una especie originaria del Hemisferio Norte: Sudeste de Europa, Anatolia, Norte de Irán y Turkestán (Paiva \& Sales, 
1990), donde también ha sido utilizada con fines medicinales (Chandra \& Rawat, 2015). Se ha naturalizado en otras regiones del mundo. En algunos países ha sido considerada maleza de cultivos y ambiental (ver citas en GCW, 2019). Hasta el momento esta especie no había sido citada como adventicia en la Argentina (Flora Argentina, 2019). Durante viajes de recolección, con el objeto de estudiar la flora del Sistema de Tandilia y las condiciones de germinación de algunas especies con valor ornamental, medicinal o de importancia biogeográfica, se localizó por primera vez una población espontánea de esta especie en las Sierras de Azul, provincia de Buenos Aires, en el año 2012. Años más tarde, en 2017, 2018 y 2019, se observó que esta población se había mantenido e incrementado su tamaño. El sitio se encuentra próximo a la Reserva Provincial "Boca de las Sierras", distante por varios kilómetros de parques y jardines rurales y a 40 $\mathrm{km}$ de la ciudad de Azul.

Se ha discutido la delimitación genérica de Lychnis L. y Silene L. (Rabeler, 1992), de modo que en floras y otros trabajos botánicos algunos autores han incluido a esta especie en el género Lychnis (Nasir \& Ali, 1988-2005; Paiva \& Sales, 1990; Oxelman et al., 2000; Wu et al., 2001; Idárraga-Piedrahita et al., 2011; Hartman \& Rabeler, 2012) y otros la han considerado perteneciente al género Silene en sentido amplio (Greuter, 1995; Morton, 2005). El Catálogo de Plantas Vasculares del Cono Sur (Zuloaga et al., 2019) la ha incorporado para Chile y Brasil con la primera denominación; en concordancia, en este trabajo se ha adoptado la misma nomenclatura. El género Lychnis se distribuye en Europa, Centro y Este de Asia, Norte y Este de África, incluye alrededor de 30 especies (Oxelman et al., 2000), varias cultivadas como ornamentales y algunas naturalizadas en otras regiones del mundo, la única para el Cono Sur y actualmente para la Argentina es $L$. coronaria. Este género pertenece a la tribu Sileneae DC., de la subfamilia Caryophylloideae. Esta subfamilia agrupa a todas las especies que presentan los sépalos unidos en gran parte de su longitud, las hojas sin estípulas, estilos libres y cápsulas pluriseminadas. La tribu Sileneae se caracteriza morfológicamente por el gineceo 3-5 carpelar y el cáliz con nervios comisurales. De los ocho géneros pertenecientes a esta tribu (Oxelman et al., 2000), sólo tres están presentes en la Argentina: Agrostemma L., Lychnis y Silene. Sólo Silene incluye algunos representantes nativos.

El cultivo de especies exóticas ornamentales y su posterior naturalización es una de las causas de invasiones biológicas (Dehnen-Schmutz et al., 2007; Dehnen-Schmutz \& Touza, 2008, van Kleunen et al., 2018). En la Argentina se han citado numerosas especies naturalizadas con ese origen y se continúan incorporando a la flora adventicia año a año nuevos registros (Delucchi et al., 2016; Cantero et al., 2017; Puntieri \& Vignoles, 2018; Scaramuzzino et al, 2018; Hurrell et al., 2019). Por lo tanto, es importante esta vía de ingreso así como también es relevante el primer registro de una especie adventicia en el contexto de las invasiones biológicas (Pyšek et al., 2004).

El objetivo del presente trabajo es citar por primera vez a $L$. coronaria para la flora adventicia argentina y aportar datos morfológicos, ambientales, fotografías de campo y un mapa con la ubicación de la población hallada. Adicionalmente, se realizó una prueba sobre la capacidad de germinación de las semillas provenientes de la población reportada. Se aporta una clave para diferenciar el género Lychnis de los restantes géneros de la tribu Sileneae que poseen representantes nativos o naturalizados en la Argentina.

\section{MATERIALES Y MÉTODOS}

El material analizado se recolectó en diciembre de 2012, diciembre de 2017, noviembre de 2018 y febrero de 2019, en Boca de las Sierras, Sierras de Azul, Sistema de Tandilia, provincia de Buenos Aires, al borde de la ruta provincial 80 y puente sobre afluente del Arroyo La Corina (3704'05.33”S - 5945'41.78”W) (Fig. 1), en un sitio próximo a la Reserva Natural Boca de las Sierras, área protegida provincial. Los ejemplares se depositaron en el herbario FAA (Thiers, 2019). El estudio morfológico se realizó bajo microscopio estereoscópico. Fue determinado con floras de diversos países y otros trabajos sobre el género y la especie (McNeill, 1978; Dimitri, 1987; Clapham et al., 1987; Paiva \& Sales, 1990; Gleason \& Cronquist, 1991; Bittrich, 1993; Tutin et al., 1993; Greuter, 1995; Morton, 2005). 
El material herborizado fue comparado con un ejemplar de la misma especie cultivado en la ciudad de Azul y depositado en el herbario FAA. Asimismo, se cotejaron los ejemplares con imágenes digitales del ejemplar tipo (http://linnean-online.org/4275/) y de otros materiales de esta especie conservados en los herbarios LINN, S, P, MO y NY.

En diciembre de 2017 se colectó y determinó la flora acompañante de esta población, y se cosecharon semillas maduras. Éstas fueron guardadas en sobres de papel a temperatura ambiente por 130 días hasta su posterior utilización en pruebas de germinación. La prueba de germinación se condujo en caja de Petri (5 repeticiones de 100 semillas cada una), usándose triple hoja de papel de filtro humedecido con agua desmineralizada, se las incubó a $23^{\circ} \mathrm{C}$ y en presencia de luz fluorescente (fotoperíodo de 8 horas). Se registró diariamente la germinación a partir de los tres y hasta los trece días de la siembra. La siembra, evaluación de la germinación y estado final de las semillas no germinadas se realizaron con el auxilio de un microscopio estereoscópico (20x).

\section{RESULTADOS}

Lychnis coronaria (L.) Desr., en Lam., Encycl. 3(2): 643. 1792. Agrostemma coronaria L. Sp. Pl. 1: 436. 1753. Silene coronaria (L.) Clairv. Man. Herbor. Suisse. 145. 1811. TIPO: "Habitat in Italia" (lectotipo LINN 601.2, designado por Ghazanfar, S. A. \& Y. J. Nasir en E. Nasir\& S. I. Ali, Fl. Pakistan, Univ. of Karachi, Caryophyllaceae 175: 55. 1986). Fig. 2.

Hierba perenne, densamente tomentosa. Tallos erectos, robustos, de aproximadamente $60-75 \mathrm{~cm}$ de altura, ramificados. Hojas opuestas, enteras, las basales en roseta, obovadas de 11-15 × 2-4 cm, base atenuada, hojas caulinares ovadas, connadas, gradualmente menores de 1,5-12 $\times 1-4,5 \mathrm{~cm}$. Inflorescencias terminales, en dicasio, laxas. Flores perfectas, conpedicelosde $1-10 \mathrm{~cm}$; cálizgamosépalo, con 10 nervios comisurales prominentes, con tubo de $1 \mathrm{~cm}$ y 5 lóbulos de $3-4 \mathrm{~mm}$; corola de $30 \mathrm{~mm}$ de diámetro, actinomorfa, pentámera, de color púrpura,

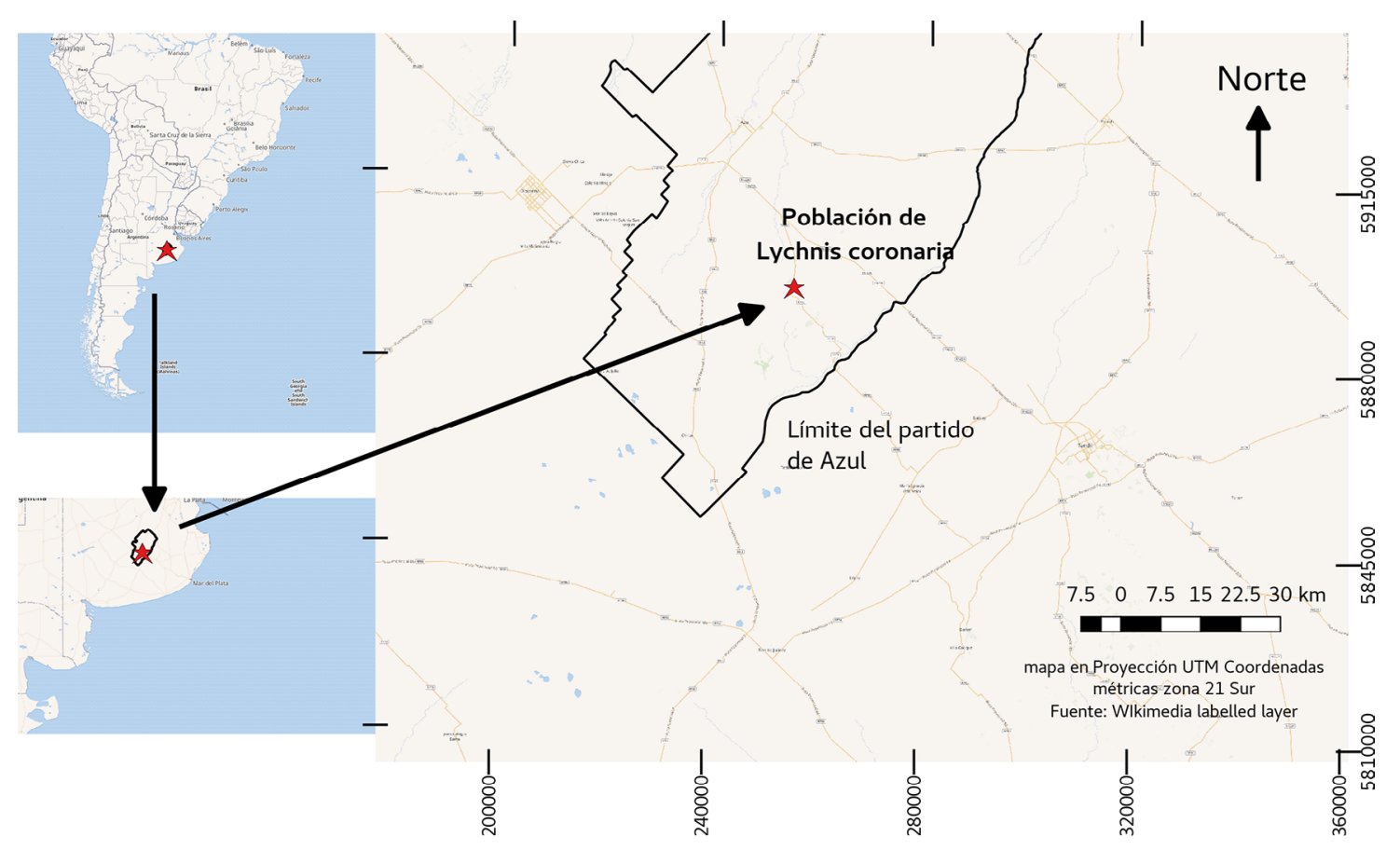

Fig. 1. Sitio de colección de Lychnis coronaria (L.) Desr. en la provincia de Buenos Aires, Argentina. Figura en color en la versión en línea http://www.ojs.darwin.edu.ar/index.php/darwiniana/article/view/826/1149 
rosado o blanco, pétalos con uña angosta, ápice del limbo redondeado o levemente emarginado, entre el limbo y la uña presenta dos escamas rígidas que forman una corona; estambres 10; ovario unilocular, pluriovulado, estilos 5, Cápsula elipsoidal, de 14-16 x 9-12 mm, pluriseminada, dehiscente por 5 dientes enteros, carpóforo de $2 \mathrm{~mm}$. Semillas de color castaño, comprimidas lateralmente, hilio en posición central, caras laterales reniformes, episperma con prominencias, con aspecto tuberculado.

Iconografía. Véase Kops et al. (1865, fig. 893), Dimitri (1987, fig. 96A) y Morton (2005).

Nombres vulgares. "oreja de gato" (Argentina), "rose campion" (inglés, Gran Bretaña, Estados Unidos de Norteamérica), "candelaria”, "clavel lanudo" (España).

Distribución y hábitat. Sudeste de Europa, Anatolia, Norte de Irán y Turkestán (Paiva \& Sales, 1990). Introducida en otros países de Europa, como España y Portugal (Paiva \& Sales, 1990), en Oceanía (Garnock-Jones, 1981), en América del Norte (Gleason \& Cronquist, 1991; Morton, 2005; Reznicek et al., 2011; Hartman \& Rabeler, 2012). En América del Sur ha sido registrada en Brasil y Chile (Baeza et al, 1999; Rodriguez et al., 2018; Zuloaga et al., 2019). En Europa como naturalizada habita en bordes y claros (Paiva \& Sales, 1990), en América del Norte, escapada de cultivo, se la encuentra a orillas de caminos, campos, bosques deciduos, áreas disturbadas, sitios rocosos (Morton, 2005; Reznicek et al., 2011; Hartman \& Rabeler. 2012). En Argentina, fue hallada en la primavera del año 2012, en Boca de las Sierras, Sierras de Azul, Sistema de Tandilia, provincia de Buenos Aires, en el borde de la ruta provincial 80, próximo al puente sobre afluente del Arroyo La Corina, cercano al Parador "Pullu Calel". En diciembre de 2017, noviembre de 2018 y febrero de 2019 se observó que esta población se había mantenido e incrementado su tamaño. Los ejemplares se ubican en ambientes rocosos y en el pastizal (Fig. 2). Entre las especies acompañantes predominaron las exóticas: Echium plantagineum L., Centaurea diffusa Lam., Lactuca saligna L., Lathyrus latifolius L., Centaurium pulchellum (Sw.) Druce, Dipsacus fullonum L., Anthoxanthum odoratum L., Bromus hordeaceus L., Phalaris aquatica L., entre otras.
Las nativas presentes fueron Bothriochloa barbinodis (Lag.) Herter, Nassella trichotoma (Nees) Hack. ex Arechav., Jarava plumosa (Spreng.) S.W.L. Jacobs \& J. Everett, Melica rigida Cav., Pavonia hastata Cav., Modiolastrum lateritium (Hook.) Krapov., Gomphrena perennis L. y otras especies frecuentes en los roquedales como Anemia tomentosa (Savigny) Sw.

Observaciones. Pyšek et al. (2004) sugieren que para poder considerar a una especie como naturalizada en una nueva región deben transcurrir al menos diez años desde su observación como espontánea. Si bien este período de tiempo no ha transcurrido en el caso de $L$. coronaria no puede clasificarse a la población estudiada como casual por varias razones. En primer lugar, la población se ha mantenido por varios ciclos de vida ya que constatamos la producción de semillas in situ en el 2017 y años posteriores. En segundo lugar, es poco probable que su persistencia se deba a reproducción por intervención humana ni a introducciones repetidas, debido al sitio donde fue hallada y a la distancia a parques y jardines. Por último, se hallaron simultáneamente plantas en distinto estado de desarrollo y la floración indica que los requerimientos absolutos de vernalización fueron cubiertos en este ambiente. La fructificación fue abundante, las semillas obtenidas resultaron altamente viables y probablemente también se reproduzca vegetativamente (Hartman \& Kester, 1986). Es posible su presencia en otros sitios dentro del Sistema de Tandilia.

En la población hallada en las Sierras de Azul, todos los individuos presentaban flores con corola blanca. Para algunos autores esto es una rareza (Paiva \& Sales, 1990; Tutin et al., 1993) pero en la Argentina, frecuentemente, se han cultivado ejemplares con esa característica (Dimitri, 1987). Al comparar los especímenes coleccionados en el ambiente serrano con un ejemplar cultivado en la ciudad de Azul se observó que el tamaño de flores y frutos fue similar pero las hojas fueron algo menores en longitud y más angostas.

Las hojas y tallos tomentosos son una característica que $L$. coronaria comparte con L. flos-jovis (L.) Desr., especie con pétalos bilobulados, que no se encuentra naturalizada ni aparentemente se cultiva en la Argentina (Dimitri, 1987) y cuya inflorescencia es diferente. Otra especie de este género, pero 
con las hojas no tomentosas, L. flos-cuculi L., ha sido citada para los alrededores de Buenos Aires, "bastante común en rastrojos de quintas y al borde de las calles de las quintas en islas del Paraná" y "en Isla Santiago, cerca de La Plata” (Spegazzini, 1905), también fue mencionada posteriormente como escapada de cultivo (Añon Suarez, 1967), pero luego fue excluida de la flora argentina (Zuloaga et al., 2019). Por lo tanto L. coronaria es la única especie de su género reportada para la Argentina.

\section{Germinación de semillas de Lychnis coronaria}

La capacidad de germinación de las semillas colectadas de la población de $L$. coronaria fue elevada $(97 \%$ en promedio con un desvío estándar $=1,87$ ) y rápida (se completó en la primera semana). La inspección de las semillas no germinadas mostró menos de un $25 \%$ de semillas blandas y/o vacías, consistiendo el $75 \%$ restante de semillas con contenido de consistencia firme y color blanco, características compatibles con un estado de dormición. En su cultivo en laboratorio no se observaron problemas fitosanitarios y las plántulas obtenidas fueron normales y completas. Estos resultados son compatibles con las observaciones sobre la germinación consignadas por Hartman y Kester (1996) y Deno (1998), en cuanto a que no requieren de tratamientos especiales para superar la dormición. Sin embargo, los tiempos obtenidos para completar la germinación fueron más cortos que las dos a cuatro semanas informadas por Hartman \& Kester (1996), como así también al tiempo medio para la germinación de casi 16 días informado por Jiang \& Dunn (2016), pero comparables con los cuatro a seis días de Deno (1998). Es poco probable que estas diferencias respondan a diferencias en las temperaturas durante la germinación $\left(20^{\circ} \mathrm{C}\right.$ en Hartman \& Kester; $18 / 20^{\circ} \mathrm{C}$ en Jiang \& Dunn y $21,1^{\circ} \mathrm{C}$ en Deno, con los $23^{\circ} \mathrm{C}$ del presente trabajo). Varias son las causas que podrían estar implicadas, una de ellas podría ser la pérdida/adquisición de dormición en relación a las condiciones y longitud del almacenamiento de las semillas y, por otro lado, a la pérdida de viabilidad en relación con longevidad de las semillas. Entre las publicaciones citadas anteriormente sólo la de Deno (1998) especifica que las semillas que germinaron en un $77 \%$ entre los cuatro a seis días habían sido almacenadas por tres años bajo temperaturas alternas $\left(4-21,1{ }^{\circ} \mathrm{C}\right)$ mientras que para las almacenadas por 4 años a temperatura constante $\left(21^{\circ} \mathrm{C}\right)$ el porcentaje obtenido a las dos semanas disminuyó a $20 \%$. Estos datos no son suficientes para realizar una comparación sólida, sin embargo, nuestros datos apuntan a que las semillas o no tienen dormición primaria o la superan con un almacenamiento en seco por menos de cuatro meses y a temperatura ambiente; mientras que por los de Deno (1998) prolongados tiempos de almacenamiento (3-4 años) estén comprometiendo la viabilidad y/o induciendo a una dormición secundaria, siendo la primera suposición más probable por tratarse de semillas de pequeño tamaño. Sin datos biológicos básicos es arriesgado hacer extrapolaciones a condiciones naturales, de todas formas y para este caso, la alta capacidad de las semillas de la población encontrada para producir plántulas normales quedó probada.

\section{Material representativo examinado}

ARGENTINA. Buenos Aires. Pdo. Azul, Boca de las Sierras, Sierras de Azul, Sistema de Tandilia, Ruta provincial $80,\left(37^{\circ} 04^{\prime} 05.33^{\prime \prime S} 59^{\circ} 45^{\prime} 41.78^{\prime \prime} \mathrm{W}\right)$, 20-XII-2012 (fl), D'Alfonso s.n. (FAA 7976), 08XII-2017 (fl), Manfreda, Gandini \& Scaramuzzino s.n.(FAA 8286), 22-XII-2017 (fr), Manfreda, Gandini, Scaramuzzino \& Alcaraz s.n. (FAA 8801), 04-XI2018 (fl), D'Alfonso s.n.(FAA 8842), 25-II-2019 (fr), Manfreda, Scaramuzzino, Alcaraz \& Acosta s.n. (FAA 9400). Material adicional (cultivado): Pdo. Azul, Azul, 13-XII-2017 (fl), Manfreda s.n. (FAA 8282).

\section{Clave de identificación de géneros de la tribu Sileneae en la Argentina}

1- Lóbulos del cáliz más largos que los pétalos Agrostemma

1- Lóbulos del cáliz más cortos que los pétalos 2

2 (1)- Cápsulas dehiscentes por 5 dientes apicales enteros. Plantas tomentosas ............................................. Lychnis

2- Cápsulas dehiscentes generalmente por 6-10 dientes apicales. Si es por 5, plantas no tomentosas Silene 

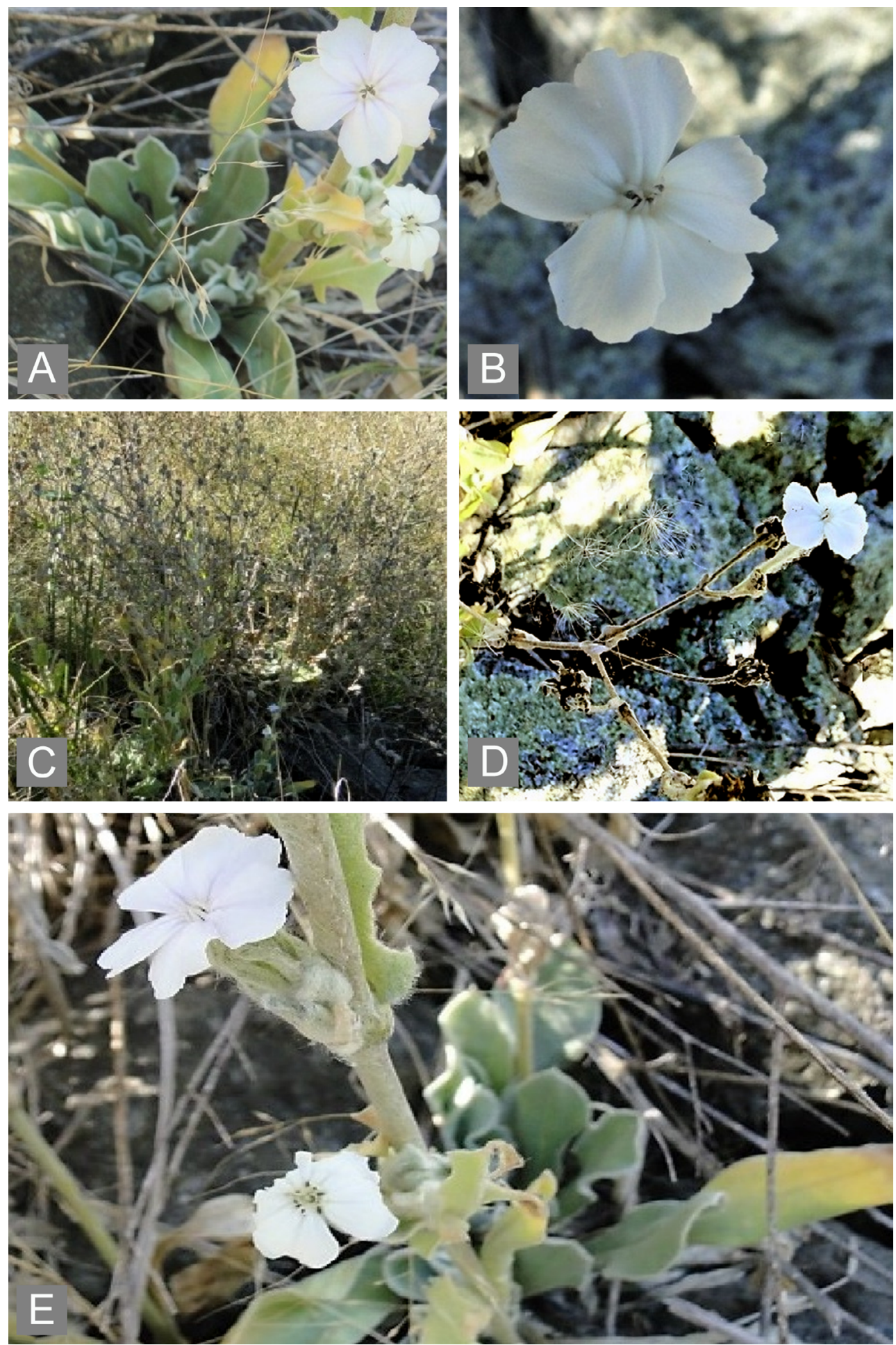

Fig. 2. Lychnis coronaria (L.) Desr. A, E, aspecto general. B, flor. C, ambiente de recolección en las Sierras de Azul, Sistema de Tandilia. D, ejemplar entre rocas. Fotografías: Carlos D’Alfonso. Figura en color en la versión en línea http://www.ojs.darwin.edu.ar/index.php/darwiniana/article/view/826/1149 


\section{R. L. SCARAMUZZINO ET AL. Lychnis coronaria en Argentina}

\section{BIBLIOGRAFIA}

Añón Suarez, D. 1967. Caryophyllaceae, en A. L. Cabrera (ed.), Flora de la provincia de Buenos Aires. Colección Cientifica Instituto Nacional de Tecnología Agropecuaria 4 (3): 192-237.

Baeza, C. M.; C. Marticorena \& R. Rodríguez Ríos. 1999. Catálogo de la flora vascular del Monumento Natural Contulmo, Chile. Gayana, Botánica 56(2): 125-135.

Bittrich, V. 1993. Caryophyllaceae, en K. Kubitzki, J. Rohwer \& V. Bittrich (eds.), The families and genera of vascular plants, volumen 2, pp. 206-236. Berlin: SpringerVerlag.

Cantero, J. J.; C. O. Núñez \& G. E. Barboza. 2017. Persicaria capitata (Polygonaceae) naturalizada en la República Argentina. Darwiniana nueva serie 5(2), 138-145. DOI: 10.14522/darwiniana.2017.52.769

Chandra, S. \& D. S. Rawat. 2015. Medicinal plants of the family Caryophyllaceae: a review of ethno-medicinal uses and pharmacological properties. Integrative Medicine Research 4(3), 123-131.

Chouard, P. 1960. Vernalization and its relations to dormancy. Annual Review of Plant Physiology 11: 191-238.

Clapham, A. R.; T. G. Tutin \& D. M. Moore. 1987. Flora of the British Isles, 3ra ed. Cambridge: Cambridge University Press.

Dehnen-Schmutz, K. \& J. Touza. 2008. Plant invasions and ornamental horticulture: pathway, propagule pressure and the legal framework, en J.A. Teixeira da Silva (ed.), Floriculture, Ornamental and Plant Biotechnology vol. 5, pp. 15-21. London: Global Science Books.

Dehnen-Schmutz, K.; J. Touza, C. Perrings \& M. Williamson. 2007. The horticultural trade and ornamental plant invasions in Britain. Conservation Biology 21: 224-231. DOI: $10.1111 /$ j.1523-1739.2006.00538.x

Delucchi, G.; N. Bayón \& J. Hurrell. 2016. Senecio angulatus (Asteraceae, Senecioneae) naturalizada en la Argentina. Boletín de la Sociedad Argentina de Botánica 51: 169-177.

Deno, N. C. 1998. Second Supplement to Seed Germination Theory and Practice. Norman C. Deno, State College Consultado en: https://naldc.nal.usda.gov/download/41277/ PDF

Dimitri, M. J. 1959. Lychnis, en L. R. Parodi (ed.), Enciclopedia Argentina de Agricultura y Jardinería, vol. 1, pp. 347-348. Buenos Aires: Ed. ACME.

Dimitri, M. J. 1987. Cariofilaceas, en L. R. Parodi (ed.), Enciclopedia Argentina de Agricultura y Jardinería, 3ra edición, actualizada por M. J. Dimitri, vol. 1, pp. 363-369; 604-605. Buenos Aires: Ed. ACME.
Flora Argentina. 2019. Flora Argentina: Plantas Vasculares de la República Argentina. Disponible en: http://www. floraargentina.edu.ar [Consulta enero 2019].

Garnock-Jones, P. J. 1981. Checklist of dicotyledons naturalized in New Zealand 8. Aizoaceae, Caryophyllaceae and Portulacaceae. New Zealand Journal of Botany 19(1): 59-65. DOI: 10.1080/0028825X.1981.10425190

GCW-Global Compendium Weeds. 2019. http://www.hear. org/gcw/species/lychnis_coronaria[Consulta marzo 2019].

Gleason, H. A. \& A. Cronquist. 1991. Manual of vascular plants of northeastern United States and adjacent Canada, 2a edición. New York: New York Botanical Garden.

Greuter, W. 1995. Silene (Caryophyllaceae) in Greece: a subgeneric and sectional classification.Taxon 44: 543581. DOI: $10.2307 / 1223499$

Hartman, H. T. \& D. E. Kester. 1986. Propagación de plantas: principios y prácticas, $6^{\mathrm{a}}$ reimpresión. México, DF: Compañía Editorial Continental.

Hartman, R. L. \& R. K. Rabeler. 2012. Lychnis coronaria, en Jepson Flora Project (eds.) Jepsone Flora: Vascular plants of California. University of California, Berkeley http:// ucjeps.berkeley.edu/eflora/ [Consulta: enero 2019].

Hurrell, J. A.; G. Delucchi \& H. A. Keller. 2019. Lilium regale (Liliaceae) naturalizada en la Argentina y nuevos registros para Brasil austral. Bonplandia (Corrientes) 28(1): 71-75. DOI: 10.30972/bon.2813574

Idárraga-Piedrahita, A.; R. D. C. Ortiz, R. Callejas Posada \& M. Merello (eds.) 2011. Flora de Antioquia: Catálogo de las Plantas Vasculares, vol. 2. Medellín: Universidad de Antioquia.

Jiang, L. \& B. L. Dunn. 2016. Ethyl Methanesulfonate and Caffeine Mutagenetic Treatment to Four Ornamental Silene Species. Journal of Environmental Horticulture 34(4): 95-100. DOI: https://www.hrijournal.org/doi/ full/10.24266/0738-2898-34.4.95

Kops, J.; P. M. E. Gevers Deijnoot \& F. A. Hartsen (eds). 1865. Flora Batava. Afbeelding en beschrijving der Nederlandschegewassen. 12. http://botanicalillustrations. org [Consulta: enero 2019]

McNeill, J. 1978. Silene alba and S. dioica in North America and the generic delimitation of Lychnis, Melandrium, and Silene (Caryophyllaceae). Canadian Journal of Botany 56 (3): 297-308. DOI: 10.1139/b78-037

Morton, J. K. 2005. Silene (Caryophyllaceae), Flora of North America Association (ed.), Flora of North America, vol. 5. http://floranorthamerica.org [Consulta: enero 2019].

Nasir, E. \& S. I. Ali (eds) 1980-2005. Flora of Pakistan. Karachi: University of Karachi. 
Oxelman, B.; M. Liden, R. K. Rabeler \& M. Popp. 2000. A revised generic classification of the tribe Sileneae (Caryophyllaceae). Nordic journal of botany 20(6): 743748. DOI: 10.1111/j.1756-1051.2000.tb00760.x

Paiva, J. \& M. F. Sales. 1990. Lychnis, en S. Castroviejo, M. Laínz, G. López González, P. Montserrat, F. Muñoz Garmendia, J. Paiva \& L. Villar (eds.), Flora Ibérica, volumen 2, pp. 299-302. Madrid: Real Jardín BotánicoConsejo Superior de Investigaciones Científicas.

Pyšek, P.; D. M. Richardson, M. Rejmánek, G.L. Webster, M. Williamson \& J. Kirschner. 2004. Alien plants in checklists and floras: towards better communication between taxonomists and ecologists. Taxon 53: 131-143. DOI: 10.2307/4135498

Puntieri, J. G. \& E. Vignoles. 2018. Buddleja davidii (Scrophulariaceae), especie invasora nueva para la flora adventicia de la Argentina. Darwiniana, nueva serie 6(1): 120-125. DOI: 10.14522/darwiniana.2018.61.789

Rabeler, R. K. 1992. (1034) Proposal to Conserve 2490 Silene L. against Lychnis L. (Caryophyllaceae). Taxon 41(1): 126128. DOI: $10.2307 / 1222505$

Rabeler, R. K. \& R. L. Hartman. 2005. Caryophyllaceae, Flora of North America Association (ed.), Flora of North America, vol. 5. http://floranorthamerica.org [Consulta: enero 2019].

Reznicek, A. A.; E. G. Voss \& B. S. Walters. 2011. Michigan Flora Online. University of Michigan, https://michiganflora.net [Consulta: enero 2019].

Rodriguez, R.; C. Marticorena, D. Alarcón, C. Baeza, L. Cavieres, V. L. Finot, N. Fuentes, A. Kiessling, M. Mihoc, A. Pauchard, E. Ruiz, P. Sanchez \& A. Marticorena. 2018. Catálogo de las plantas vasculares de Chile. Gayana, Botánica 75(1): 1-430.
Scaramuzzino, R.; V. Manfreda \& M. Gandini. 2018. Verbascum Blattaria (Scrophulariaceae): primer registro para la República Argentina. Darwiniana, nueva serie 6(1): 126-132. DOI: 10.14522/darwiniana.2018.61.802

Spegazzini, C. L. 1905. Flora de la provincia de Buenos Aires. Buenos Aires: Anales del Ministerio de Agricultura, sección de Biología Vegetal.

Thiers, B. [permanentemente actualizado, consulta 2019] Index Herbariorum: a global directory of public herbaria and associated staff. New York Botanical Garden's Virtual Herbarium, http://sweetgum.nybg.org/ih

Tutin, T. G.; N. A. Burges, A. O. Chater, J. R. Edmondson, V. H. Heywood, D. M. Moore, D. H. Valentine, S. M. Walters \& D. A. Webb (eds.) 1993. Flora Europaea, 2da edición, vol. 1. Cambridge: Cambridge University Press.

Van Kleunen, M.; F. Essl; J. Pergl, G. Brundu, M. Carboni, S. Dullinger, R. Early, P. González-Moreno, Q. J. Groom, P. E. Hulme, C. Kueffer, I. Kühn, C. Máguas, N. Maurel, A. Novoa, M. Parepa, P. Pyšek, H. Seebens, R. Tanner, J. Touza, L. Verbrugge, E. Weber, W. Dawson, H. Kreft, P. Weigelt, M. Winter, G. Klonner, M. V. Talluto \& K. Dehnen-Schmutz. 2018. The changing role of ornamental horticulture in alien plant invasions. Biological Reviews 93(3):1421-1437. DOI: 10.1111/brv.12402

Wu, C.; P. H. Raven \& D.Y. Hong (eds.) 2001. Flora of China. Beijing \& St. Louis: Science Press \& Missouri Botanical Garden Press.

Zuloaga, F.; O. Morrone \& M. J. Belgrano. 2019 Catálogo de las plantas del Cono Sur. Base de datos permanentemente actualizada y disponible en: http://www2.darwin.edu.ar/ [Consulta: enero 2019]. 